\title{
GOBERNANZA URBANA: NUEVOS RETOS Y CLAVES EN EL CONTEXTO DEL DESARROLLO URBANO SOSTENIBLE
}

\author{
Prof. a Dra. MARÍA LUISA GÓMEZ JIMÉNEZ \\ Directora WPS RI-SHUR
}

Cerramos el año 2016, con un número especial y monográfico dedicado a la Gobernanza Urbana ${ }^{1}$, que viniera a incorporar las contribuciones realizadas en el I Seminario sobre Gobernanza Urbana, que tuvo lugar el 25 de noviembre de 2016 en el Seminario de García de Enterría de Derecho Administrativo de la Facultad de Derecho de la Universidad Complutense de Madrid.

La actividad organizada en la Red Temática sobre Desarrollo Urbano Sostenible, (URBANRED) $^{2}$, de la que el Grupo de Investigación Pasos Vivienda forma parte, tenía por objeto, examinar desde la atención al desarrollo urbano sostenible las nuevas dinámicas de la Gobernanza incorporar procesos participativos en la toma de decisiones como forma de desarrollar una capacidad para concitar consenso, o, al menos, un significativo apoyo social. Para ello, se requiere la presencia activa del conjunto de la comunidad en la identificación de los problemas y soluciones, como así, en las etapas de ejecución, administración y control de gestión de las acciones en el ámbito de lo público. En esta nueva cultura de gobernanza aparece un eje central y motor de la participación y la gestión de lo público: las tecnologías.

Este es pues un número que plantea retos, difunde resultados de investigación y permite no sólo la generación de nuevas estrategias y aproximaciones a los contextos del desarrollo urbano sostenible sino que aboga por mantener la interdisciplinariedad aunque matizada en esta ocasión por la visión que se aporta desde el Derecho Administrativo, las políticas públicas y el urbanismo.

\footnotetext{
${ }^{1}$ El presente monográfico es una publicación de la Red Temática de Desarrollo Urbano, URBAN RED, proyecto enmarcado en la Convocatoria 2015 de Acciones de dinamización "Redes de Excelencia", del Programa Estatal de Investigación Científica y Técnica de Excelencia, Subprograma Estatal de Generación de Conocimiento, en el marco del Plan Estatal de Investigación Científica y Técnica y de Innovación 20132016 (Resolución de 17 de junio de 2015, de la Secretaría de Estado de Investigación, Desarrollo e Innovación, Ministerio de Economía y Competitividad, BOE 23 de junio 2015). Proyecto: DER2015-71345REDT. En cumplimiento con lo dispuesto en el artículo 6 de la citada Resolución se hace constar que la publicación de cada una de los artículos que integran el mismo ha sido posible gracias a la financiación de Ministerio de Economía y Competitividad en cofinanciación con fondos FEDER. 2 http://www.unioviedo.es/Desarrollosostenible/urban-red/index.php

${ }^{2}$ http://www.unioviedo.es/Desarrollosostenible/urban-red/index.php
}

WPS RI-SHUR, nำ, 2016, vol.1, ISSN: 2387-1768

NÚMERO MONOGRÁFICO SOBRE GOBERNANZA URBANA/SPECIAL ISSUE ON GOVERNANCE 
El número, respeta la tradicional diferenciación entre propuestas de actuación y parámetros de sostenibilidad y lo hace, con la ambigua percepción de que alguno de los trabajos seleccionados podrían encajar perfectamente en ambas categorías. No integra el apartado de Voces de los Expertos que se publica en cada número impar de la revista, y se suma por ello mayor contenido a cada una de las secciones.

Así, en la sección de Parámetros de Sostenibilidad, se integran los trabajos de los Profesores Dra. Fátima E. Ramallo López, profesora de Derecho Administrativo del Grupo de Investigación, Economía Urbana para el desarrollo Sostenible, de la Universidad de Granada. La Dra. Ramallo dedica su artículo al examen del derecho a la ciudad, buen Gobierno y buena Administración. Su trabajo, nos recuerda el carácter de función administrativa del desarrollo urbano sostenible, y los retos que dicha consideración implican especialmente para necesaria coordinación y armonización pública.

Es ésta una temática que conecta con la que el Prof. Dr. Omar Bouzza Ariño, Profesor Titular de Derecho Administrativo de la Universidad Complutense de Madrid, nos plantea al examinar la participación ciudadana en el proceso planificador. El Prof. Bouzza, es miembro del grupo de Investigación, "Transformaciones del Estado y la autonomía local: organización institucional, servicios públicos y democracia participativa”, que dirige el Prof. Dr. Tomás Cano Campos, desde el área de Derecho Administrativo en la Universidad Complutense de Madrid.

No podía ser de otra forma pues, la atención a la sostenibilidad- como carácter a tomar presente en el análisis de lo urbano se proyecta también en la sostenibilidad social, económica, amén de la ambiental. Así, el Catedrático de Derecho Administrativo de la Universidad de Girona Dr. José Manuel Trayter Jiménez, que dirige el Grup de Recerca de Dret Urbanistic, centra su contribución en el examen de la planificación territorial y urbanística, y plantea propuestas y retos, del urbanismo tomando como telón de fondo la interconexión entre la ordenación del territorio, el urbanismo y la vivienda. Nos recuerda el autor: "El Plan debe crear ciudades que proporcionen un entorno atractivo para sus habitantes mediante la técnica de la planificación urbanística y de la mezcla de usos". Ciertamente ahí se encuentra la esencia de la mejora de la calidad de vida que permita una adecuada sostenibilidad Social.

\footnotetext{
${ }^{3}$ https://www.ucm.es/grupos/grupo/245
}

WPS RI-SHUR, n4, 2016, vol.1, ISSN: 2387-1768

NÚMERO MONOGRÁFICO SOBRE GOBERNANZA URBANA/SPECIAL ISSUE ON GOVERNANCE 
No podría ser de otra forma, como señala la Directora de la Cátedra UniversidadEmpresa de Accesibilidad en la Edificación, de la Universidad Politécnica de Madrid e Investigadora Colaboradora del Grupo de Investigación Pasos Vivienda, Dr. Nieves Navarro Cano. La profesora Navarro Cano, incorpora en su contribución, la proyección de la cohesión social a través de la atención a la ciudad sostenible como ciudad accesible. Atiende su trabajo a los aspectos vinculados a la diversidad funcional de colectivos cuyo número se incrementará en los años venideros, que deben convivir en una ciudad habitable.

Así las cosas, se cierra el bloque de Parámetros de Sostenibilidad, con las contribuciones de los investigadores Dr. Moneyba González Molina, de la Universidad Autónoma de Madrid, la Dra. María Ángeles Huete García, y el Dr. Rafael Merinero Rodríguez de la Universidad de Universidad Pablo de Olavide, con un artículo que nos trae reflexiones sobre la agenda urbana de la Unión europea, y el examen de los hitos más significativos de su evolución con proyección histórica. Examen que permite resituarnos en el contexto actual, y prever las posibles estrategias de desarrollo de una agenda Urbana, que, si bien- en construcción - resulta de suma importancia para las actuaciones públicas en nuestro país. Son justamente estas actuaciones que se proyectan con distinta suerte en la geografía española, sobre las que se abordan aspectos específicos y se hacen propuestas en la segunda parte del Monográfico.

En esta segunda parte se incorpora el trabajo del Prof. Dr. Miguel Carrero de la Roa, arquitecto del Grupo de Estudios Jurídico-Sociales sobre Territorio y Desarrollo Sostenible (GDTS), que dirige la Catedrática de Derecho Administrativo de la Universidad de Oviedo, Dra. María Rosario Alonso lbáñez (directora de la red temática URBAN RED). En dicha contribución se analizan las nuevas formas de Gobernanza metropolitana en el área central de Asturias, con la detección de mejoras y propuestas de actuación que impliquen incidir en una gobernanza metropolitana sostenible.

Es desde este escenario de la proyección en la gobernanza local, desde el que se desarrolla también la intervención que propone el Catedrático de Derecho Administrativo de la Universidad de Granada, director del Grupo de Investigación sobre Economía Urbana para el Desarrollo Sostenible, y del Centro Iberoamericano de Gobernabilidad, Políticas y Administración Pública, el Dr. Federico Castillo Blanco, en el trabajo que nos aporta y que titula: "La reforma de los gobiernos locales en España: ¿una oportunidad perdida para lograr una planta local competitiva y sostenible?", y es que, como nos plantea el autor: la reforma local : "no ha ido acompañada ni de una reflexión suficiente sobre el sistema competencial local, ni tampoco, se diga lo que diga, sobre la organización local, ni tampoco sobre el sistema de dirección política y directiva de nuestros Gobiernos locales". Es desde estas premisas desde la que se incorporan reflexiones de enorme interés para el Gobierno local que

WPS RI-SHUR, n4, 2016, vol.1, ISSN: 2387-1768

NÚMERO MONOGRÁFICO SOBRE GOBERNANZA URBANA/SPECIAL ISSUE ON GOVERNANCE 
nos resitúan de nuevo en las propuestas de actuaciones de gobernanza, planteando alternativas y sugiriendo actuaciones.

Así la cosas, llegamos al trabajo del Catedrático de Derecho Administrativo de la Universidad de las Islas Baleares, Dr. Avelino Blasco Esteve; la Profesora Dra. Joana Socias Camacho, titular de Derecho Administrativo, Dr. Bartomeus Trias Prats, y la Dra. Aina Salom Parets, que desde el grupo de Investigación, Autonomía, Medio Ambiente, Turismo y Territorio, que dirige el Prof. Blasco Esteve en las Islas Baleares, nos proponen examinar el caso del Plan de reconversión de la Playa de la Palma, desde el reto que supone integrar nuevas formas de gobernanza del espacio urbano. Reflexiones que evocan la significación del contexto en el que se operan y la población a la que van dirigida las actuaciones urbanas, y que permiten conectar inclusive con la diferente cultura jurídica que internacionalmente puedan apreciarse.

Por ello, concluye este monográfico con una contribución que se asoma a la comparativa con el derecho norteamericano, y lo hace a la luz del examen de otra cuestión innovadora que conecta con la gobernanza. Este es el caso del trabajo que nos aporta la profesora Dra. Teresa Parejo Navajas, en relación a la conexión entre gobernanza y la noción de bienes comunes en una economía colaborativa que la lleva a plantear la noción de ciudad compartida. Un concepto este de ciudad compartida que resuena con el eco de las últimas investigaciones en materia de economía del bien común, y que se deben entender en el escenario de un desarrollo urbano sostenible. La Dra. Parejo por lo demás forma parte del Instituto Pascual Madoz del Territorio, Urbanismo y Medio Ambiente, de la Universidad Carlos III de Madrid, bajo la dirección, del Catedrático de Derecho Administrativo Dr. Luciano Parejo Alfonso.

WPS RI-SHUR, acoge pues, esta edición con el entusiasmo que genera ser participe en un momento de construcción científica y académica significativa, que viene a reflejar el "status quo" del debate sobre gobernanza urbana sostenible, convirtiéndose así en el vehículo oficial de difusión de las actividades académicas de la Red temática de excelencia URBAN RED.

Confiamos que su contenido sea de especial interés a nuestros lectores y les invitamos a participar y sumarse al debate que estos temas plantean.

En Málaga a 30 de diciembre de 2016

WPS RI-SHUR, n4, 2016, vol.1, ISSN: 2387-1768

NÚMERO MONOGRÁFICO SOBRE GOBERNANZA URBANA/SPECIAL ISSUE ON GOVERNANCE 